\section{EL PROCESO DE BOLONIA Y LA INTEGRACIÓN DE LA ENSEÑANZA MILITAR EN EL SISTEMA EDUCATIVO GENERAL: UN NUEVO PASO}

\author{
Juan Romero Serrano \\ General de División del Ejército de Tierra \\ Subdirector General de Enseñanza de la Dirección General \\ de Reclutamiento y Enseñanza Militar (DIGEREM) entre \\ 2006 y junio de 2012 \\ ieee@oc.mde.es
}

\begin{abstract}
Cómo citar este artículo/Citation: Romero Serrano, J. (2014). "El Proceso de Bolonia y la integración de la enseñanza militar en el sistema educativo general: un nuevo paso". Arbor, 190 (765): a100. doi: http://dx.doi.org/10.3989/ arbor.2014.765n1007
\end{abstract}

Recibido: 5 junio 2012. Aceptado: 2 diciembre 2013.

RESUMEN: El trabajo describe la reforma que introduce en la enseñanza de formación de los cuadros de mando, la "Ley de la carrera militar", integrando esta plenamente en el sistema educativo general, gracias a requerir que los oficiales obtengan un título oficial de graduado y los suboficiales de técnico superior de formación profesional, sin que suponga merma de su formación castrense, gracias a que los títulos que se cursan contribuyen a la formación necesaria para el ejercicio de la profesión militar.

PALABRAS CLAVE: Proceso de Bolonia; Ley de la carrera militar; formación militar.

\section{THE BOLOGNA PROCESS AND INTEGRATION OF MILITARY TEACHING IN THE GENERAL EDUCATION SYSTEM: A NEW STEP}

Copyright: (c) 2014 CSIC. Este es un artículo de acceso abierto distribuido bajo los términos de la licencia Creative Commons Attribution-Non Commercial (by-nc) Spain 3.0.
ABSTRACT: This paper describes the reform introduced in the education and training of officers, the "military career law," integrating it fully into the general educational system by requiring officers to obtain an official degree and non-commissioned officers to have technical training. This entails no loss of their military training, as the qualifications and titles obtained contribute to the training they need to pursue a military career.

KEYWORDS: Bologna Process; military career law; military career. 


\section{INTRODUCCIÓN}

Las personas que componen las Fuerzas Armadas se organizan en cuerpos a los que se les encomienda, mediante normativa, diferentes cometidos en orden al correcto funcionamiento de la Institución.

Se estructuran en tres tipos de escalas: oficiales, suboficiales y tropa y marinería. En este trabajo nos vamos a referir a la reforma implantada en la enseñanza de formación que da acceso a las diferentes escalas de oficiales y suboficiales de los cuerpos generales del Ejército de Tierra, la Armada y el Ejército del Aire (en adelante, los ejércitos) y del cuerpo de Infantería de Marina.

Con esta reforma se logra unificar el criterio según el cual, para acceder a los empleos de oficial es preciso un título oficial universitario, bien sea de licencia$\mathrm{do}^{1}$, graduado (a partir de la reforma de Bolonia) o diplomado. Por analogía, se establece el mismo criterio para las escalas de suboficiales, siendo preciso para el acceso obtener un título oficial de técnico superior de formación profesional.

Este trabajo no intenta entrar en las razones de fondo sobre la conveniencia del cambio, ni en los procesos de estudio y decisión entre diferentes alternativas, etc., trata de una aproximación al modelo a través de la descripción de sus componentes.

\section{EL PROCESO DE BOLONIA}

El llamado proceso de Bolonia ha sido el eje sobre el cual se ha construido el Espacio Europeo de Educación Superior (EEES), proyecto en el que se encuentran involucrados más de 40 países europeos. Muestra a su vez, vocación de relacionarse con otros espacios de educación superior, para responder a la presencia creciente del fenómeno de la globalización.

Su objetivo es la adopción de un sistema de titulaciones comprensible y comparable, permitiendo a la vez una amplia flexibilidad en los currículos. Conlleva cambios importantes en el actual sistema educativo universitario. Estos cambios están orientados a facilitar la movilidad profesional y geográfica que impone la creciente integración europea, gracias a diseños semejantes en la enseñanza superior y a criterios de transparencia y comparabilidad, de modo que se pueda saber, en función de los estudios realizados, las capacidades que cada persona aporta con bastante propiedad. Todo ello con una apuesta por fundamentar su acreditación en sistemas de garantía de la calidad.

En consecuencia se ha rediseñado el sistema educativo español en su nivel universitario, dando lugar a una reforma de gran calado para la Universidad española que se podría resumir en lo siguiente:

- Adopción de los nuevos ciclos de Grado, Master y Doctor.

- Orientación de la enseñanza hacia el aprendizaje del alumno.

- Titulaciones comparables, gracias a los perfiles de egreso expresados en competencias, con validez en todo el EEES.

- Flexibilización en las ofertas educativas (Planes de estudio).

- Establecimiento del sistema de créditos "ECTS" como medida.

- Validación de las titulaciones mediante procesos de garantía de calidad.

- Formación a lo largo de toda la vida (LLL).

- Importancia de los idiomas.

De forma paralela, y por los mismos motivos, ha sido preciso ir adaptando y actualizando los estudios de los ciclos de formación profesional, revalorizándose la preparación y capacidades que ofrecen teniendo en cuenta su relación con el tejido productivo de referencia (autonómico o nacional).

\section{ANTECEDENTES: EL SISTEMA DE ENSEÑANZA MILITAR}

El modelo vigente hasta el curso 2010-2011 de enseñanza de formación ${ }^{2}$ de los oficiales y suboficiales, nace de la reforma que se introduce en la Ley 17/1989, reguladora del Régimen del Personal Militar Profesional y se consolida en la siguiente Ley 17/1999, de Régimen del Personal de las Fuerzas Armadas. En esencia se establece un "sistema de enseñanza militar" que, siendo propio de las Fuerzas Armadas, se diseña en paralelo al sistema educativo general y adopta sus mismos parámetros. En dichas leyes el modelo militar aparece bajo el concepto de "integrado" en el sistema general debido a que se construye de manera plenamente comparable.

Las enseñanzas se desarrollaban en centros propios, a cargo de cuadros de profesores militares, casi en su totalidad, que impartían tanto asignaturas y materias de contenido genuinamente militar, como aquellas que son compartidas con el ámbito universitario y con la formación profesional. Estas asignaturas y materias compartidas son plenamente necesarias para sustentar las primeras ${ }^{3}$ y contribuyen a lograr una adecuada formación humana y técnica. 
Las directrices generales de estos planes de estudios daban lugar a currículos que contaban con una estructura semejante a la que se establecía para las carreras universitarias y de formación profesional, con iguales cargas lectivas. Estas semejanzas permitieron que la Ley $17 / 1999$, en su artículo 51, estableciera unas equivalencias genéricas entre los niveles académicos de licenciado, diplomado y técnico superior y los primeros empleos militares adquiridos en el momento de incorporarse a las escalas Superior de Oficiales, de Oficiales y de Suboficiales, respectivamente.

Como consecuencia de los paralelismos mencionados, ya en los trabajos de desarrollo de la Ley de la ca-

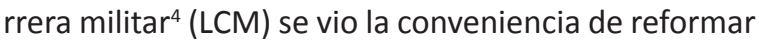
el sistema de enseñanza, para adecuarlo a las reformas que se estaban afrontando en los niveles académicos del sistema educativo general. También se valoró la oportunidad de dar un paso más en su proceso de integración en el sistema educativo general, que sería a la postre, su inclusión en los nuevos espacios europeos de enseñanza, permitiendo disfrutar de sus ventajas a los estudiantes militares.

\section{LA REFORMA: DE QUÉ SE TRATA}

Así, la Ley de la carrera militar logra la plena integración de la enseñanza que se imparte a los componentes de las Fuerzas Armadas en el sistema educativo general. El aspecto fundamental en que se concreta este nuevo paso es que los oficiales y suboficiales cursarán ${ }^{5}$ una titulación oficial, de grado en el primer caso y de formación profesional, títulos de Técnico Superior, en el segundo. Hay que señalar que el Marco Español de Cualificaciones para la Educación Superior $^{6}$ (MECES) identifica estos dos niveles académicos como el primero y segundo de la Educación Superior.

La reforma se situó, por lo tanto, ante el nuevo reto que implicaba transitar desde enseñanzas propias y específicas de las Fuerzas Armadas, a enseñanzas en las que se integran plenamente la formación militar y enseñanzas oficiales del propio sistema educativo general, con lo que todo ello implicaba.

Dichos títulos oficiales del sistema educativo general han sido escogidos teniendo en cuenta el criterio de los diferentes ejércitos y atendiendo al principio de simplicidad ${ }^{7}$. Requisito imprescindible ha sido su contribución a los cometidos y funciones que deben desempeñar los oficiales ${ }^{8}$ y suboficiales en el ámbito profesional.

La profesión militar, y las responsabilidades que se asumen en los primeros empleos de las diferentes es- calas de oficiales y de suboficiales, requieren en cada caso un núcleo de competencias específicas, exclusivas y propias de la profesión, a la vez que comparten otras competencias con otros ámbitos de conocimiento. Las cualidades que identifican con mayor claridad la profesión militar, y le otorgan ese carácter distintivo, se articulan en torno a competencias correspondientes a los planos del "saber ser" y del "saber hacer". Evidentemente el "corpus de conocimientos", que configuran el plano del "saber", contiene materias y asignaturas específicas del saber profesional militar, como no podía ser de otra forma, pero también es verdad que el "saber militar", se basa en otras disciplinas básicas, bien sean las ciencias puras, las naturales o las sociales.

Es, como digo, en los planos del saber ser y hacer donde la profesión militar requiere una serie de competencias específicas en mayor grado si se compara con la mayoría de las profesiones. Hay que tener presente que la profesión militar se desarrolla -en sus momentos más genuinos- en ambientes de gran incertidumbre, donde la información de la situación no es clara, e incluso está desvirtuada (los engaños del enemigo) y en condiciones de peligro personal y colectivo, donde hay que decidir y actuar, normalmente, contra el tiempo, con serenidad pero con rapidez.

\section{LA NUEVA ENSEÑANZA. LÍNEAS MAESTRAS}

La implantación de este modelo, en el caso de los oficiales, se llevó a cabo en el curso 2010-2011, y en el caso de los suboficiales se inició en el siguiente curso escolar ${ }^{9}$. Las líneas directrices de la reforma se pueden resumir en las siguientes:

Se proporcionan en el mismo tiempo "dos titulaciones" ${ }^{10}$ una militar y una de grado universitario o de técnico superior de formación profesional.

- Se suprime en el concurso oposición para el acceso, las clásicas pruebas de conocimientos adicionales. El reconocimiento médico, las pruebas físicas y la de nivel de inglés solo dan apto o no apto.

- La selección se hace siguiendo los criterios universitarios o de formación profesional para el acceso a los centros respectivos en cada caso. Es decir, ordenando los aspirantes según su nota de admisión ${ }^{11}$.

- El fracaso escolar y cualquier otro tipo de baja en el proceso formativo, es asumido con el incremento en el número de plazas de ingreso y con el ingreso directo con titulación previa. 
- La selección se produce de forma natural durante los años de formación.

- Se certifican a aquellos que no deseen o no puedan continuar sus estudios en los centros militares de formación, los estudios acreditados universitarios (créditos ECTS) y de formación profesional (materias y asignaturas) que hayan obtenido los alumnos, de modo que puedan continuar sus estudios sin partir de cero.

\section{CONSERVANDO LO ESENCIAL DEL "SER" EN LA FORMACIÓN MILITAR}

En esta transformación de profundo calado, se pretende preservar los aciertos de la formación impartida hasta la reforma, ampliamente contrastados por el quehacer de las Fuerzas Armadas Españolas en ambientes internacionales y en contextos de trabajo que han requerido una profunda colaboración con fuerzas amigas de diferentes países.

Para lograrlo se ha mantenido la formación de los futuros cuadros de mando en los centros castrenses tradicionales, las Academias y Escuelas. En ellos se produce la transmisión y la interiorización de los hábitos de comportamiento que presiden la forma de conducirse de los miembros en la organización militar, en especial el conjunto de "Reglas de comportamiento del militar" que la Ley Orgánica 9/2011, de 27 de julio, de derechos y deberes de los miembros de las Fuerzas Armadas establece en su artículo 6. En estas Reglas se pueden identificar tres vertientes: la primera permite al militar adaptarse a la organización, la segunda permite a la organización desenvolverse y alcanzar sus objetivos y en tercer lugar se contribuye con estos a metas generales del Estado.

Para lograr que la lealtad, la disciplina, el valor, etc. formen parte de la forma profesional de actuación de los futuros oficiales y suboficiales, es necesario que se plasmen y vivencien tanto en las clases en aula, como en las clases prácticas de campo. El ejemplo de sus profesores militares y su vida académica diaria crean un ambiente en el que su práctica sea plena.

En este sentido, si bien se ha producido un determinado cambio en el conjunto de asignaturas y materias que se imparten y, muy especialmente, su valor como materias universitarias o del ciclo superior de la formación profesional, el régimen de vida de los alumnos es básicamente el mismo que el que regulaba tradicionalmente la vida académica de las diferentes promo- ciones. Es decir, la vida de los alumnos se desenvuelve y se desenvolverá en unos ambientes presididos por una exigente disciplina militar, un comportamiento regido por el resto de las virtudes militares, y por una convivencia estrecha entre los componentes de la promoción, y de esta con las anteriores y posteriores, que fomenta el sentido de unidad, el compañerismo y la colaboración.

\section{EL ALUMNO, DESDE SU INGRESO, ES MILITAR}

El aspirante a ingresar en los centros docentes militares de formación debe reunir unos requisitos generales (LCM - Art. 56) y deben superar unas pruebas de carácter físico, médico y psicológico, que aseguren que están en condiciones de poder cursar los planes de estudio militares sin riesgo para su salud y en buenas condiciones.

Desde el momento de su ingreso en las Academias y Escuelas adquieren la condición militar y, en consecuencia, se integra en un centro en el que el régimen de vida está orientado a que pueda desarrollar sus planes de estudios y adquirir de forma completa, las competencias (valores, conocimientos, habilidades y destrezas) previstas en su perfil de salida.

\section{LAS DESTREZAS Y HABILIDADES PRÁCTICAS: EL SABER HACER}

El segundo factor que se conserva en la nueva enseñanza de formación, es su vertiente práctica. Las Academias y Escuelas tradicionalmente han venido combinando de forma adecuada las enseñanzas teóricas en aula, con las prácticas. La formación de la persona, no persigue solo orientar y facilitar un desarrollo intelectual y técnico, y la capacidad de aplicarlo, sino que se persigue en todos los casos un desarrollo armónico e integral.

Elemento relevante de esta vertiente teórico-práctica de la formación de los oficiales y suboficiales es la materia que en los términos militares se denomina Instrucción y Adiestramiento, y que no es otra cosa que las sesiones prácticas para integrar conocimientos provenientes de diferentes asignaturas (organización, táctica, logística, etc.) en actividades diseñadas como un todo y que tienen por finalidad el aprendizaje paulatino del ejercicio de la profesión.

Esta buena combinación se complementaba con una formación física orientada fundamentalmente a promover hábitos saludables, el necesario desarrollo corporal a través del ejercicio y el deporte y el endurecimiento en tan importante etapa de la vida del joven. 


\section{LOS NUEVOS PLANES DE ESTUDIOS}

La LCM establece que es condición imprescindible para el acceso a las diferentes escalas de oficiales y suboficiales la obtención previa de un título de grado universitario o de técnico superior de formación profesional.

Para lograr esto se ha recurrido a dos modelos muy semejantes pero que tienen alguna diferencia relevante.

El modelo de oficiales se diseña para que durante cinco cursos escolares se impartan las dos enseñanzas: la específicamente militar y la de grado. Ya se ha comentado antes que la elección de las titulaciones a impartir está guiada por la contribución que tenga el plan de estudios universitario a la formación de base del futuro oficial, todo ello de acuerdo con la idea también comentada de que el "saber" militar se nutre y se asienta en buena medida en el saber general distribuido en diferentes campos. El tipo de formación, más general ${ }^{12}$, que se busca en los oficiales, nos permite elegir un número reducido de titulaciones de grado en cada Cuerpo.

El esquema se aproxima mucho a lo que, hasta ahora, se entendía en España como una "doble titulación", es decir, cursar dos carreras con currículos complementarios integrando estos en un plan de estudios único. El resultado de elegir unas titulaciones de grado que incluyeran buena parte de los antiguos planes de estudio militares ${ }^{13}$ ha permitido integrar los nuevo planes y desplegarlos en cinco cursos escolares intensos, con un número elevado de créditos por curso $(\mathrm{ECTS})^{14}$, que se logran superar gracias a las condiciones de internado en que se encuentran los alumnos, grupos de clase pequeños, tutorización muy personalizada y todos aquellos recursos para facilitar que el estudiante se centre en su importante cometido.

En el caso de los suboficiales el esquema general es el mismo. Sin embargo, los cometidos que llevan a cabo estos profesionales, que se agrupan según su "especialidad fundamental" (infantería, automoción, informática, transmisiones, mantenimiento de aeronaves, etc.) en la mayoría de los casos tienen una correspondencia directa con profesiones civiles. Por lo tanto, se diseña el sistema escogiendo para cada especialidad fundamental de las escalas de suboficiales el título de técnico superior adecuado. Así, el modelo exige que se impartan entre 15 y 20 titulaciones de formación profesional frente al corto número de las titulaciones de grado. Dichos planes de estudios se entretejen con los planes específicamente militares, en un ambiente plenamente militar en sus respectivas Escuelas y Academias, logrando que su formación se complete en tres cursos escolares ${ }^{15}$ con sus prácticas en talleres, laboratorios y campo de maniobras.

Reuniendo las competencias en los tres planos descritos se obtiene el "perfil de egreso" o salida del futuro oficial o suboficial. La responsabilidad de fijar dichos perfiles la tiene el respectivo Jefe de Estado Mayor del ejército correspondiente que decide así a través de las competencias qué oficial o suboficial requiere el respectivo ejército. Para comprobar que dichos perfiles se obtienen, y en qué medida, y corregir las posibles desviaciones, se está ensayando un sistema de evaluación específico para este fin con la inestimable colaboración de la Agencia Nacional de Evaluación de la Calidad y Acreditación (ANECA).

\section{DOS CENTROS DIFERENTES: MILITAR Y UNIVERSITARIO}

¿Dónde se imparten estos planes de estudios integrados? Al diseñar el modelo se pensó que el carácter de la formación no aconsejaba impartir los planes de estudios secuencialmente, sino que era preferible atender a su carácter de integrados y desplegarlos simultáneamente. En ese esquema no era eficiente que el alumno estuviera desplazándose de un centro a otro y se vio la necesidad de diseñar la estructura de centros de modo que fuera lo mejor para el alumno.

En el caso de los oficiales, las enseñanzas universitarias son impartidas por los centros universitarios de la defensa (CUD) ${ }^{16}$ que se configuran como un sistema que pudiera crecer, si se sintiera esa necesidad. Al principio se crearon tres centros ubicados en la Academia General Militar ${ }^{17}$ (Zaragoza) en la Escuela Naval Militar $^{18}$ (Marín, Pontevedra) y en la Academia General del Aire ${ }^{19}$ (San Javier, Murcia).

Estos centros adquieren la condición de universitarios gracias a los Convenios de Adscripción entre el Ministerio de Defensa y las Universidades de Zarago$z a$, Vigo y Politécnica de Cartagena ${ }^{20}$. Así se convierten en centros de las citadas universidades, en su modalidad de adscritos:

\section{"Cuando culmine el proceso de adscripción de los centros, estos tendrán naturaleza de centro universi- tario público, adscrito a la universidad correspondien- te, con la denominación de centro universitario de la defensa" ${ }^{21}$.}

En consecuencia, sus actividades docentes e investigadoras están sujetas a las correspondientes autorizaciones ("venias docendi", etc.) y supervisión de los órganos de gobierno de aquellas y de los organismos 
encargados de supervisar los procesos de verificación, acreditación y garantía de calidad (ANECA o agencias autonómicas autorizadas).

Como resultado de todos estos controles, el Rector correspondiente expedirá los títulos oficiales de grado y posgrado que se impartan en dichos centros.

En el caso de los suboficiales, las mismas Academias y Escuelas que impartían las enseñanzas para la incorporación a las escalas de suboficiales, son los centros que recibieron la autorización del Ministerio de Educación, Cultura y Deporte para impartir los planes de estudio conducentes a la obtención de los títulos de Técnico Superior. Ha sido necesario acomodar las instalaciones, laboratorios y talleres a los requisitos que en cada caso se precisaban para poder obtener la autorización del ministerio de Educación, Cultura y Deporte ${ }^{22}$, así como disponer de los profesores civiles necesarios aportados por las consejerías de educación de las Comunidades Autónomas ${ }^{23}$ en las que se radican las Escuelas y Academias donde se imparten los títulos de formación profesional.

\section{LOS PROFESORES}

Las titulaciones civiles se van a impartir por profesores que reúnan los requisitos necesarios. En el caso de los oficiales, se emplean diferentes modalidades. Desde el profesor contratado por el centro y que debe obtener la "venia docendi" del Rector de la universidad de adscripción, hasta el profesor funcionario docente en virtud de convenio o en servicios especiales.

En el caso de los suboficiales la docencia se podrá impartir por profesores militares o civiles, ambos tipos con los requisitos necesarios de titulación y capacidad docente, fundamentalmente. Los profesores civiles, tal como se acaba de comentar, gracias a la aportación de las Comunidades Autónomas, mediante profesores funcionarios en comisión de servicio o interinos contratados.

\section{CONFIGURACIÓN Y PUESTA EN MARCHA}

Con lo que se ha comentado hasta aquí, se puede decir que los dos modelos -ambos muy similaresestán configurados y en marcha. Los pilares son los clásicos: los alumnos, su razón de ser, los planes de estudio y régimen de vida académica, los profesores y la estructura de centros.

Este próximo julio finaliza el tercer curso del modelo de oficiales y el segundo del de suboficiales. Los primeros síntomas de cómo responde el modelo de oficiales en este cambio, se han obtenido del proce- so selectivo para ingresar en las diferentes escalas de oficiales. La respuesta de los jóvenes está siendo muy positiva, multiplicando por 2.5 el número de aspirantes del año 2010, año de su implantación, respecto al año 2009 y manteniéndose esa acogida positiva en los siguientes, lo que demuestra que el modelo, que conlleva su titulación universitaria de grado, es atractivo a un número bastante más elevado de aspirantes.

Se ha logrado una igualdad ante la opción de ingreso al no ser preciso "preparar" una oposición muy específica, a cargo de centros no oficiales dedicados a ese cometido, y que hacía prácticamente imprescindible acudir a alguno de ellos, con el consiguiente gasto de matrícula, más el añadido de tener que residir en una población en la que hubiera. Cualquier joven español, en cualquier punto de España, puede solicitar su ingreso en uno de los tres ${ }^{24}$ centros de formación militares ${ }^{25}$.

Por otra parte, como ya se ha mencionado, el criterio tenido en cuenta para seleccionar a los que han ingresado, de entre aquellos que finalmente superaron las pruebas médicas, psicológicas, físicas y de nivel mínimo de inglés, ha sido su nota de admisión a la universidad. Este procedimiento ha establecido unas notas de corte elevadas, que traducidas a la tradicional escala de 0 a 10, se sitúan en el entorno del 7 para el alumno con menor nota de los tres centros universitarios de la defensa.

Esta nota de corte alta asegura que la calidad de los alumnos es la adecuada para afrontar unos planes de estudio exigentes, en contextos diferentes y nuevos para los alumnos, que demuestran una intensa ilusión aceptando y superando las dificultades, importante semilla para perfeccionar su vocación militar, imprescindible en esta profesión u "oficio", como gusta decir a los clásicos.

Está claro que las experiencias y los resultados de estos primeros cursos proporcionarán las pautas para hacer los ajustes precisos para que el modelo integrado de doble titulación funcione mejor. Se partió de la plena confianza en los componentes de los centros militares y universitarios implicados.

En el caso de los oficiales, la Academia General Militar, la Escuela Naval Militar y la Academia General del Aire, son centros de excelencia, con una experiencia muy amplia, que están sabiendo articular los diferentes elementos del modelo de forma cada vez más eficiente, respondiendo a una apuesta de futuro, en la que están implicados tanto los Ejércitos de Tierra, la Armada y el Ejército del Aire, como el propio Ministerio de Defensa, desde donde se han organizado e impulsando los dos modelos. 
1 Licenciado, ingeniero o arquitecto.

2 Ya se ha anotado que es la enseñanza que da acceso a las respectivas escalas.

3 No se podría estudiar sistemas radáricos sin saber la suficiente electrónica, informática y electricidad.

4 Ley 39/2007, de 19 de noviembre, de la carrera militar.

5 Como ya se ha apuntado al principio, en todo el artículo se trata de los oficiales y suboficiales de los Cuerpos Generales del Ejército de Tierra, la Armada y el Ejército del Aire y el Cuerpo de Infantería de Marina. Los demás oficiales de las Fuerzas Armadas ya ingresan con su titulación universitaria correspondiente.

6 Real Decreto 1027/2011, de 15 de julio, por el que se establece el Marco Español de Cualificaciones para la Educación Superior. Una estructura en cuatro niveles que debe permitir situar a una persona según su nivel de aprendizaje adquirido y certificado.

7 Incorporar en el caso de los oficiales inicialmente un título de grado por ejército para no complicar excesivamente la implantación de la reforma. En el caso de los suboficiales, dada su mayor especialización y por ser más numerosos los centros de enseñanza, se ha optado por un número más elevado de titulaciones de formación profesional de grado superior.

8 Los tres ejércitos se inclinaron por la rama de las ingenierías.

9 Como excepción a lo establecido en la Introducción, en el caso de los oficiales, para los oficiales médicos del Cuerpo Militar de Sanidad, se implantó el modelo de ingreso sin titulación oficial previa de médico, para que se cursara dicho título en las Fuerzas Armadas, en el curso 2012-2013.

10 El correspondiente Real Despacho y e Título oficial de graduado o de Técnico superior.
11 La nota de admisión de cada aspirante al ingreso se conforma según lo establecido como criterio por el sistema educativo general. Es cierto que se puede ver ligeramente aumentada en determinados casos por considerar como mérito pertenecer previamente a las Fuerzas Armadas.

12 De hecho los oficiales se agrupan en los Cuerpos Generales y su especialización inicial tiende a difuminarse frente a la enseñanza del s. XX en el que predominaban las tradicionales Armas: Infantería, Caballería, Artillería e Ingenieros, herederas de las Armas Generales y los Cuerpos Facultativos del s. XIX.

13 Los posibles ejemplos son numerosos pero sirvan de muestra materias como las Matemáticas, la Informática para la Ingeniería, la Teoría de máquinas y mecanismos, la Topografía, la Electrónica, el Liderazgo, la Dirección de recursos humanos, el inglés, etc.

$14 \mathrm{Ha}$ sido necesario sobrepasar los 60 ECTS por curso escolar para responder a unos planes de estudio más exigentes, sin que ello resulte un criterio que se pueda considerar excesivo.

15 Evidentemente, cuando se habla de cinco o tres cursos escolares, se trata del tiempo en el que se despliegan los planes de estudio integrados. Otra cosa es el tiempo que necesite cada alumno para superarlos y lograr su Despacho Real y su título oficial y así, acceder a la escala correspondiente.

16 http://cud.unizar.es/, http://cud.uvigo. es/, http://www.cud.upct.es/

17 http://www.ejercito.mde.es/unidades/ Zaragoza/agm/

18 http://www.armada.mde.es/ArmadaPortal/page/Portal/ArmadaEspannola/personal_escuelas/prefLang_es/02_enm

19 http://www.ejercitodelaire.mde.es/ea/ pag?idDoc=FBACC6B47954912CC1257 ODD00429B2C\&idRef=06C72C57F949F B01C1257459002649FB
20 Resolución 420/01910/2010, de 29 de enero, de la Secretaría General Técnica, por la que se da publicidad al Convenio de adscripción entre el Ministerio de Defensa y la Universidad de Zaragoza para regular las condiciones conforme a las cuales el centro universitario de la defensa en la Academia General Militar se adscribe a la Universidad de Zaragoza. (Están las tres resoluciones seguidas en el mismo BOD, núm. 25 de 8 de febrero de 2010).

21 Creados por REAL DECRETO 1723/2008, de 24 de octubre, por el que se crea el sistema de centros universitarios de la defensa. Este RD ha sido modificado y ampliado, entre otras cosas, para dar cabida al modelo de formación de médicos militares.

22 Orden EDU/3602/2011, de 12 de diciembre, por la que se autoriza la implantación de enseñanzas de formación profesional en varios centros docentes militares a partir del año 2012. (BOE núm. 2, del 3 de enero de 2012.

23 Gracias a los correspondientes convenios entre cada Comunidad Autónoma, el Ministerio de Educación y el de Defensa.

24 Actualmente cuatro, con el de los médicos militares, en este artículo solo referenciado. EI CUD correspondiente se ubica en el Grupo de Escuelas de la Defensa (Madrid) y está adscrito a la Universidad de Alcalá. Imparte el Grado en Medicina con el apoyo de la Escuela Militar de Sanidad y el Hospital Central de la Defensa “Gómez Ulla”.

25 Para el ingreso en la Escala Superior de Oficiales de la Guardia Civil se usa el sistema del Ejército de Tierra, cursando los dos primeros años en la Academia general Militar de Zaragoza. 